\title{
On Nonautonomous Discrete Dynamical Systems
}

\author{
Dhaval Thakkar ${ }^{1}$ and Ruchi Das ${ }^{2}$ \\ ${ }^{1}$ Vadodara Institute of Engineering, Kotambi, Vadodara 391510, India \\ ${ }^{2}$ Department of Mathematics, Faculty of Science, The M.S. University of Baroda, Vadodara 390002, India
}

Correspondence should be addressed to Ruchi Das; rdasmsu@gmail.com

Received 26 November 2013; Accepted 18 April 2014; Published 2 June 2014

Academic Editor: Harumi Hattori

Copyright (C) 2014 D. Thakkar and R. Das. This is an open access article distributed under the Creative Commons Attribution License, which permits unrestricted use, distribution, and reproduction in any medium, provided the original work is properly cited.

We define and study expansiveness, shadowing, and topological stability for a nonautonomous discrete dynamical system induced by a sequence of homeomorphisms on a metric space.

\section{Introduction}

In the recent past, lots of studies have been done regarding dynamical properties in nonautonomous discrete dynamical systems. In [1], Kolyada and Snoha gave definition of topological entropy in nonautonomous discrete systems. In [2], Kolyada et al. discussed minimality of nonautonomous dynamical systems. In [3, 4], authors studied $\omega$-limit sets in nonautonomous discrete systems, respectively. In [5], Krabs discussed stability and controllability in nonautonomous discrete systems. In $[6,7]$, Huang et al. studied topological pressure and preimage entropy of nonautonomous discrete systems. In [8-15], authors studied chaos in nonautonomous discrete systems.

In [16], Liu and Chen studied $\omega$-limit sets and attraction of nonautonomous discrete dynamical systems. In $[8,17]$ authors studied weak mixing and chaos in nonautonomous discrete systems. In [18] Yokoi studied recurrence properties of a class of nonautonomous discrete systems. Recently in [19] we defined and studied expansiveness, shadowing, and topological stability in nonautonomous discrete dynamical systems given by a sequence of continuous maps on a metric space.

In this paper we define and study expansiveness, shadowing, and topological stability in nonautonomous discrete dynamical systems given by a sequence of homeomorphisms on a metric space. In the next section, we define and study expansiveness of a time varying homeomorphism on a metric space. In section following to the next section, we define and study shadowing or P.O.T.P. for a time varying homeomorphism on a metric space. In the final section, we study topological stability of a time varying homeomorphism on a compact metric space.

\section{Expansiveness of a Nonautonomous Discrete System Induced by a Sequence of Homeomorphisms}

Throughout this paper we consider $(X, d)$ to be a metric space and $f_{n}: X \rightarrow X$ to be a sequence of homeomorphisms, $n=$ $0,1,2, \ldots$, where we always consider $f_{0}$ to be the identity map on $X$ and $F=\left\{f_{n}\right\}_{n=0}^{\infty}$ to be a time varying homeomorphism on $X$. We denote

$$
F_{n}= \begin{cases}f_{n} \circ f_{n-1} \circ \cdots \circ f_{1} \circ f_{0}, & \text { for } n \geq 0 \\ f_{-n}^{-1} \circ f_{-(n-1)}^{-1} \circ \cdots \circ f_{1}^{-1} \circ f_{0}^{-1} & \text { for } n \leq-1 .\end{cases}
$$

For any $0 \leq i \leq j$, we define

$$
F_{[i, j]}=f_{j} \circ f_{j-1} \circ \cdots \circ f_{i+1} \circ f_{i},
$$

and, for $i>j$, we define $F_{[i, j]}$ to be the identity map on $X$. For time varying homeomorphism $F=\left\{f_{n}\right\}_{n=0}^{\infty}$ on $X$, its inverse 
map is given by $F^{-1}=\left\{f_{n}^{-1}\right\}_{n=0}^{\infty}$. For any $k>0$, we define a time varying map ( $k$ th-iterate of $F) F^{k}=\left\{g_{n}\right\}_{n=0}^{\infty}$ on $X$, where

$$
\begin{array}{r}
g_{n}=f_{n k} \circ f_{(n-1) k+k-1} \circ \cdots \circ f_{(n-1) k+2} \circ f_{(n-1) k+1} \\
\forall n \geq 0 .
\end{array}
$$

Thus $F^{k}=\left\{F_{[(n-1) k+1, n k]}\right\}_{n=0}^{\infty}$, for $k>0$, and, for $k=-m<0$, $F^{k}=\left(F^{-1}\right)^{m}$. Also, for $k=0, F^{k}=\left\{f_{n}\right\}_{n=0}^{\infty}$, where each $f_{n}$ is the identity map on $X$.

Definition 1. Let $(X, d)$ be a metric space and $f_{n}: X \rightarrow X$ a sequence of maps, $n=0,1,2, \ldots$. For a point

$x_{0} \in X$, let

$$
x_{n}= \begin{cases}f_{n}\left(x_{n-1}\right) & n \geq 1 \\ f_{-n}^{-1}\left(x_{n+1}\right) & n \leq-1\end{cases}
$$

then the sequence $\left\{x_{n}\right\}_{n=-\infty}^{\infty}$, denoted by $O\left(x_{0}\right)$, is said to be the orbit of $x_{0}$ under time varying homeomorphism $F=$ $\left\{f_{n}\right\}_{n=0}^{\infty}$.

Definition 2. Let $(X, d)$ be a metric space and $f_{n}: X \rightarrow X$ a sequence of homeomorphisms, $n=0,1,2, \ldots$. The time varying homeomorphism $F=\left\{f_{n}\right\}_{n=0}^{\infty}$ is said to be expansive if there exists a constant $e>0$ (called an expansive constant) such that, for any $x, y \in X, x \neq y, d\left(F_{n}(x), F_{n}(y)\right)>e$ for some $n \in \mathbb{Z}$. Equivalently, if, for $x, y \in X, d\left(F_{n}(x), F_{n}(y)\right) \leq e$ for all $n \in \mathbb{Z}$ then $x=y$.

Remark 3. If in the above definition $f_{n}=f$, for all $n \geq 0$, where $f: X \rightarrow X$ is homeomorphism, then expansiveness of time varying homeomorphism $F=\left\{f_{n}\right\}_{n=0}^{\infty}$ on $X$ is equivalent to expansiveness of $f$ on $X[20]$.

Remark 4. Note that expansiveness of a time varying homeomorphism $F$ is independent of the choice of metric for $X$ if $X$ is compact.

Definition 5. Let $\left(X, d_{1}\right)$ and $\left(Y, d_{2}\right)$ be two metric spaces. Let $F=\left\{f_{n}\right\}_{n=0}^{\infty}$ and $G=\left\{g_{n}\right\}_{n=0}^{\infty}$ be time varying homeomorphisms on $X$ and $Y$, respectively. If there exists a homeomorphism $h: X \rightarrow Y$ such that $h \circ f_{n}=g_{n} \circ h$ for all $n=0,1,2, \ldots$ then $F$ and $G$ are said to be conjugate with respect to the map $h$ or $h$-conjugate. In particular, if $h$ : $X \rightarrow Y$ is a uniform homeomorphism, then $F$ and $G$ are said to be uniformly conjugate or uniformly $h$-conjugate. (Recall that homeomorphism $h: X \rightarrow Y$, such that $h$ and $h^{-1}$ are uniformly continuous, is called a uniform homeomorphism.)

For example, if $F=\left\{x^{n+1}\right\}_{n=0}^{\infty}$ on $[0,1]$ and $G=\{2((x+$ $\left.1) / 2)^{n+1}-1\right\}_{n=0}^{\infty}$ on $[-1,1]$ are time varying homeomorphisms, then $F$ is uniformly $h$-conjugate to $G$, where $h:[0,1] \rightarrow$ $[-1,1]$ is defined by $h(x)=2 x-1$.

Theorem 6. Let $\left(X, d_{1}\right)$ and $\left(Y, d_{2}\right)$ be metric spaces. Let $F=$ $\left\{f_{n}\right\}_{n=0}^{\infty}$ and $G=\left\{g_{n}\right\}_{n=0}^{\infty}$ be time varying homeomorphisms on $X$ and $Y$, respectively, such that $F$ is uniformly conjugate to $G$. Then $F$ is expansive on $X$ if and only if $G$ is expansive on $Y$.
Proof. Since $F$ is uniformly conjugate to $G$, therefore there exists a uniform homeomorphism $h: X \rightarrow Y$ sach that $h \circ f_{n}=g_{n} \circ h$, for all $n \geq 0$, which implies that $f_{n} \circ h^{-1}=$ $h^{-1} \circ g_{n}$, for all $n \geq 0$, and $f_{n}^{-1} \circ h^{-1}=h^{-1} \circ g_{n}^{-1}$, for all $n \geq 0$. Now, for all $n \geq 0$,

$$
\begin{aligned}
F_{n} \circ h^{-1}= & f_{n} \circ f_{n-1} \circ \cdots f_{2} \circ f_{1} \circ f_{0} \circ h^{-1} \\
= & f_{n} \circ f_{n-1} \circ \cdots f_{2} \circ f_{1} \circ h^{-1} \circ g_{0} \\
& \vdots \\
= & h^{-1} \circ g_{n} \circ g_{n-1} \circ \cdots g_{2} \circ g_{1} \circ g_{0} \\
= & h^{-1} \circ G_{n},
\end{aligned}
$$

and similarly, for all $n \leq 0$, we also have

$$
\begin{aligned}
F_{n} \circ h^{-1}= & f_{-n}^{-1} \circ f_{-n+1} \circ \cdots f_{2}^{-1} \circ f_{1}^{-1} \circ f_{0}^{-1} \circ h^{-1} \\
= & f_{-n}^{-1} \circ f_{-n+1}^{-1} \circ \cdots f_{2}^{-1} \circ f_{1}^{-1} \circ h^{-1} \circ g_{0}^{-1} \\
& \vdots \\
= & h^{-1} \circ g_{n}^{-1} \circ g_{-n+1}^{-1} \circ \cdots g_{2}^{-1} \circ g_{1}^{-1} \circ g_{0}^{-1} \\
= & h^{-1} \circ G_{n} .
\end{aligned}
$$

So we get $F_{n} \circ h^{-1}=h^{-1} \circ G_{n}$, for all $n \in \mathbb{Z}$. Similarly, $h \circ$ $F_{n}=G_{n} \circ h$, for all $n \in \mathbb{Z}$. Suppose $F$ is expansive on $X$ with expansive constant $\varepsilon>0$. Since $h^{-1}$ is uniformly continuous, therefore there exists a $\delta>0$ such that, for any $y_{1}, y_{2} \in Y$ with $d_{2}\left(y_{1}, y_{2}\right)<\delta, d_{1}\left(h^{-1}\left(y_{1}\right), h^{-1}\left(y_{2}\right)\right)<\varepsilon$. Let $y_{1}, y_{2} \in Y$ such that $y_{1} \neq y_{2}$; then $h^{-1}\left(y_{1}\right) \neq h^{-1}\left(y_{2}\right)$ and since $F$ is expansive on $X$; there exists $n \in \mathbb{Z}$ such that

$$
\begin{aligned}
& d_{1}\left(h^{-1}\left(G_{n}\left(y_{1}\right)\right), h^{-1}\left(G_{n}\left(y_{2}\right)\right)\right) \\
& \quad=d_{1}\left(F_{n}\left(h^{-1}\left(y_{1}\right)\right), F_{n}\left(h^{-1}\left(y_{2}\right)\right)\right)>\varepsilon,
\end{aligned}
$$

which implies that $d_{2}\left(G_{n}\left(y_{1}\right), G_{n}\left(y_{2}\right)\right) \geq \delta$. Hence $G$ is expansive on $Y$.

Conversely, suppose $G$ is expansive on $Y$ with expansive constant $\varepsilon>0$. Since $h$ is uniformly continuous, there exists $\delta>0$ such that, for any $x_{1}, x_{2} \in X$ with $d_{1}\left(x_{1}, x_{2}\right)<\delta$, $d_{2}\left(h\left(x_{1}\right), h\left(x_{2}\right)\right)<\varepsilon$. For any $x_{1}, x_{2} \in X$ with $x_{1} \neq x_{2}$, observing that $h\left(x_{1}\right) \neq h\left(x_{2}\right)$, it follows that there exists $n \in \mathbb{Z}$ such that

$$
\begin{aligned}
d_{2}( & \left.\left(F_{n}\left(x_{1}\right)\right), h\left(F_{n}\left(x_{2}\right)\right)\right) \\
& =d_{2}\left(G_{n}\left(h\left(x_{1}\right)\right), G_{n}\left(h\left(x_{1}\right)\right)\right)>\epsilon,
\end{aligned}
$$

which implies that $d_{1}\left(F_{n}\left(x_{1}\right), F_{n}\left(x_{2}\right)\right) \geq \delta$. Thus $F$ is expansive on $X$.

Corollary 7. Let $\left(X, d_{1}\right)$ be a compact metric space, $\left(Y, d_{2}\right)$ a metric space, $F=\left\{f_{n}\right\}_{n=0}^{\infty}$ a time varying homeomorphism on $X$, and $h: X \rightarrow Y$ a homeomorphism. If $F$ is expansive on $X$, then $G=h \circ F \circ h^{-1}=\left\{h \circ f_{n} \circ h^{-1}\right\}_{n=0}^{\infty}$ is expansive on $Y$. 
Theorem 8. Let $(X, d)$ be a compact metric space and $\left\{f_{n}\right\}_{n=0}^{\infty}$ a family of self-homeomorphisms on $X$. Then time varying map $F=\left\{f_{n}\right\}_{n=0}^{\infty}$ is expansive if and only if $F^{-1}$ is expansive.

Proof. Let $e>0$ be an expansive constant for $F$. It is easy to verify that $F_{(-n)}=\left(F^{-1}\right)_{n}$, for all $n \in \mathbb{Z}$. Let $x \neq y, x, y \in X$; then there is some $n \in \mathbb{Z}$ such that $d\left(F_{n}(x), F_{n}(y)\right)>e$; that is, $d\left(\left(F^{-1}\right)_{(-n)}(x),\left(F^{-1}\right)_{(-n)}(y)\right)>e$, for some $n \in \mathbb{Z}$, which implies $F^{-1}$ is also expansive.

Using the above Theorem, analogous to Theorem 2.2 in [19], we have the following result.

Theorem 9. Let $(X, d)$ be a compact metric space, $\left\{f_{n}\right\}_{n=0}^{\infty}$ an equicontinuous family of self-maps on $X$, and $k$ an integer. Then time varying homeomorphism $F=\left\{f_{n}\right\}_{n=0}^{\infty}$ is expansive if and only if $F^{k}$ is expansive for any $k \in \mathbb{Z}-\{0\}$.

Definition 10. Let $(X, d)$ be a metric space, $F=\left\{f_{n}\right\}_{n=0}^{\infty}$ a time varying homeomorphism on $X$, and $Y$ a subset of $X$. Then $Y$ is said to be invariant under $F$ if $f_{n}(Y)=Y$ (and therefore $f_{n}^{-1}(Y)=Y$ ), for all $n \geq 0$, and equivalently $F_{n}(Y)=Y$, for all $n \in \mathbb{Z}$.

We have the following result from the definition of invariance.

Theorem 11. Let $(X, d)$ be a metric space, $F=\left\{f_{n}\right\}_{n=0}^{\infty}$ a time varying homeomorphism which is expansive on $X$, and $Y$ an invariant subset of $X$; then restriction of $F$ to $Y$, defined by $F \mid$ $Y=\left\{f_{n} \mid Y\right\}$, is expansive.

Similar to Theorem 2.4 in [19], we have the following result.

Theorem 12. Let $\left(X, d_{1}\right)$ and $\left(Y, d_{2}\right)$ be metric spaces and $F=$ $\left\{f_{n}\right\}_{n=0}^{\infty}, G=\left\{g_{n}\right\}_{n=0}^{\infty}$ time varying homeomorphisms on $X$ and $Y$, respectively. Consider the metric $d$ on $X \times Y$ defined by

$$
\begin{array}{r}
\left(\left(x_{1}, y_{1}\right),\left(x_{2}, y_{2}\right)\right)=\max \left\{d_{1}\left(x_{1}, x_{2}\right), d_{2}\left(y_{1}, y_{2}\right)\right\} ; \\
\left(x_{1}, y_{1}\right),\left(x_{2}, y_{2}\right) \in X \times Y .
\end{array}
$$

Then the time varying homeomorphism $F \times G=\left\{f_{n} \times g_{n}\right\}_{n=0}^{\infty}$ is expansive on $X \times Y$ if and only if $F$ and $G$ are expansive on $X$ and $Y$, respectively. Hence every finite direct product of expansive time varying homeomorphisms is expansive.

We have the following result for time varying homeomorphism similar to that for expansive homeomorphism on compact metric space [21].

Theorem 13. Let $(X, d)$ be a compact metric space and $F=$ $\left\{f_{n}\right\}_{n=0}^{\infty}$ a time varying homeomorphism which is expansive on $X$. If $\theta$ is the least upper bound of the expansive constants for $F$, then $\theta$ is not an expansive constant for $F$.

Proof. Let $e$ be an expansive constant for $F$ and $0<\theta<$ $e$; then $\theta$ is also an expansive constant for $F$. Let $\varepsilon_{i}=$ $1 / i$, for $i=1,2,3, \ldots$. Since $\theta+\varepsilon_{i}$ is not an expansive constant for $F$, therefore for each $i$ there exist $x_{i}^{\prime} \neq y_{i}^{\prime}$ such that
$d\left(F_{n}\left(x_{i}^{\prime}\right), F_{n}\left(y_{i}^{\prime}\right)\right) \leq \theta+\varepsilon_{i}$ for each integer $n$. Also, for each $i$, there exists an integer $k_{i}$ such that $d\left(F_{k_{i}}\left(x_{i}^{\prime}\right), F_{k_{i}}\left(y_{i}^{\prime}\right)\right)>e$. Let $x_{i}=F_{k_{i}}\left(x_{i}^{\prime}\right)$ and $y_{i}=F_{k_{i}}\left(y_{i}^{\prime}\right)$. We can assume that the sequences $\left\{x_{i}\right\}_{i=0}^{\infty}$ and $\left\{y_{i}\right\}_{i=0}^{\infty}$ converge to $x$ and $y$, respectively, and we note that $x \neq y$. Let $m$ be an arbitrary integer and $\alpha$ an arbitrary positive number. Choose $p, q$, and $\eta$ with the following properties. (1) $\varepsilon_{p}<\alpha / 3$, (2) $d(u, v)<\eta$ implies $d\left(F_{n}(u), F_{n}(v)\right)<\alpha / 3$, and (3) $n>p$ implies $d\left(x, x_{n}\right)<\eta$ and $n>q$ implies $d\left(y, y_{n}\right)<\eta$. Let $i>\max \{p, q\}$; then

$$
\begin{aligned}
d\left(F_{m}(x), F_{m}(y)\right) \leq & d\left(F_{m}(x), F_{m}\left(x_{i}\right)\right) \\
& +d\left(F_{m}\left(x_{i}\right), F_{m}\left(y_{i}\right)\right) \\
& +d\left(F_{m}\left(y_{i}\right), F_{m}(y)\right) \\
\leq & \frac{\alpha}{3}+\theta+\frac{\alpha}{3}+\frac{\alpha}{3}=\alpha+\theta .
\end{aligned}
$$

Thus $d\left(F_{m}(x), F_{m}(y)\right) \leq \theta$ and therefore $\theta$ is not an expansive constant for $F$.

The topological analogue of generator was defined and studied by Keynes and Robertson [22]. We define and study this notion for invertible nonautonomous discrete dynamical system.

Definition 14. Let $(X, d)$ be a compact metric space and $F=$ $\left\{f_{n}\right\}_{n=0}^{\infty}$ a time varying homeomorphism on $X$. A finite open cover $\alpha$ of $X$ is said to be a generator for $F$ if, for every bisequence $\left\{A_{n}\right\}$ of members of $\alpha, \bigcap_{n=-\infty}^{\infty}\left(F_{n}\right)^{-1}\left(\overline{A_{n}}\right)$ is at most one point, where $\overline{A_{n}}$ denotes the closure of $A_{n}$.

Definition 15. Let $(X, d)$ be a compact metric space and $F=$ $\left\{f_{n}\right\}_{n=0}^{\infty}$ a time varying homeomorphism on $X$. A finite open cover $\alpha$ of $X$ is said to be a weak generator for $F$ if for every bisequence $\left\{A_{n}\right\}$ of members of $\alpha, \bigcap_{n=-\infty}^{\infty}\left(F_{n}\right)^{-1}\left(\overline{A_{n}}\right)$ is at most one point.

Theorem 16. Let $(X, d)$ be a compact metric space and $F=$ $\left\{f_{n}\right\}_{n=0}^{\infty}$ a time varying homeomorphism on $X$. Then the following are equivalent.

(1) $F$ is expansive.

(2) F has a generator.

(3) F has a weak generator.

Proof. (2) $\Rightarrow$ (3) follows by definitions of generator and weak generator. We prove that $(3) \Rightarrow(2)$. Let $\beta=\left\{B_{1}, B_{2}, \ldots, B_{n}\right\}$ be a weak generator for $F$ and $\delta>0$ a Lebesgue number for $\beta$. Let $\alpha$ be a finite open cover by sets $A_{i}$ with $\operatorname{diam}\left(\overline{A_{i}}\right) \leq \delta$. If $\left\{A_{i_{n}}\right\}$ is a bisequence of members of $\alpha$, then for every $n$ there is $j_{n}$ such that $\overline{A_{i_{n}}} \subset B_{j_{n}}$, and so $\bigcap_{-\infty}^{\infty}\left(F_{n}\right)^{-1}\left(\overline{A_{i_{n}}}\right) \subset$ $\bigcap_{-\infty}^{\infty}\left(F_{n}\right)^{-1}\left(B_{j_{n}}\right)$. Since $\bigcap_{-\infty}^{\infty}\left(F_{n}\right)^{-1}\left(B_{j_{n}}\right)$ contains almost one point, therefore $\bigcap_{-\infty}^{\infty}\left(F_{n}\right)^{-1}\left(\overline{A_{i_{n}}}\right)$ also contains atmost one point and hence $\alpha$ is a generator.

Next we prove that $(1) \Rightarrow(2)$. Let $\delta>0$ be an expansive constant for $F$ and $\alpha$ a finite open cover of $X$ by open balls of radius $\delta / 2$. Suppose $x, y \in \bigcap_{-\infty}^{\infty}\left(F_{n}\right)^{-1}\left(\overline{A_{i_{n}}}\right)$, where $A_{i_{n}} \in \alpha$; 
then $d\left(F_{n}(x), F_{n}(y)\right) \leq \delta$ for every $n$ and since $F$ is expansive with expansive constant $\delta$, we have $x=y$.

$(3) \Rightarrow(1)$ : suppose $\alpha$ is a weak generator. Let $\delta>0$ be a Lebesgue number for $\alpha$. If $d\left(F_{n}(x), F_{n}(y)\right)<\delta$, for all $n \in$ $\mathbb{Z}$, then for every $n$ there is $A_{n} \in \alpha$ such that $F_{n}(x), F_{n}(y) \in$ $A_{n}$ and so $x, y \in \bigcap_{-\infty}^{\infty}\left(F_{n}\right)\left(A_{n}\right)$ which is at most one point implying $x=y$.

Example 17. Let $F=\left\{f_{n}\right\}_{n=0}^{\infty}$, where $f_{n}:[0,1] \rightarrow[0,1]$, such that $f_{n}(x)=x^{n+1}$, for $n=0,1,2 \ldots$ and $x \in[0,1]$, be a time varying homeomorphism on $[0,1]$. Now note that $F_{n}(x)=x^{n !}$ and $F_{-n}=x^{1 / n !}$ for all $n \geq 0$. Let $\alpha$ be a finite open cover of $[0,1]$ with Lebesgue's number $0<\delta<1 / 2$. Note that $\lim _{n \rightarrow \infty} F_{n}=0$ and $\lim _{n \rightarrow \infty} F_{-n}=1$ uniformly on $[\delta, 1-\delta]$. Then there exists $N>0$ such that $n>N$ implies $F_{n}(x) \in[0, \delta)$ and $F_{-n}(x) \in(1-\delta, 1]$, for any $x \in[\delta, 1-\delta]$. Since $\delta$ is Lebesgue's number of $\alpha$, there are $A_{0}$ and $A_{1}$ in $\alpha$ such that $[0, \delta) \subset A_{0}$ and $(1-\delta, 1] \subset A_{1}$. Now since $\left\{F_{-N}, F_{-N+1}, \ldots, F_{N}\right\}$ is uniformly equicontinuous family, there exists $\varepsilon>0$ such that $d(x, y)<\varepsilon$ implies $d\left(F_{n}(x), F_{n}(y)\right)<\delta$, for any $|n| \leq N$. Let $x, y \in[\delta, 1-\delta]$, $x \neq y$, such that $d(x, y)<\varepsilon$. Then, for any $n,|n| \leq N$ there exists $A_{n} \in \alpha$ such that $F_{n}(x), F_{n}(y) \in A_{n}$. Thus $x, y \in$ $\left(F_{n}\right)^{-1}\left(A_{n}\right),|n| \leq N$. Now put

$$
A_{n}= \begin{cases}A_{0}, & n \geq N+1 \\ A_{1}, & n \leq-(N+1) .\end{cases}
$$

Now note that

$$
x, y \in \bigcap_{n=-\infty}^{\infty}\left(F_{n}\right)^{-1}\left(A_{n}\right) .
$$

Thus $\alpha$ cannot be a weak generator for $F$. Therefore $F$ has no weak generator and hence by the above result $F$ is a nonexpansive time varying homeomorphism.

\section{Pseudo Orbit Tracing Property of a Nonautonomous Discrete System by a Sequence of Homeomorphisms (P.O.T.P.)}

Definition 18. Let $(X, d)$ be a metric space and $F=\left\{f_{n}\right\}_{n=0}^{\infty}$ a time varying homeomorphism on $X$. For $\delta>0$, the sequence $\left\{x_{n}\right\}_{n=-\infty}^{\infty}$ in $X$ is said to be $a \delta$-pseudo orbit of $F$ if

$$
\begin{aligned}
& d\left(f_{n+1}\left(x_{n}\right), x_{n+1}\right)<\delta, \quad \text { for } n \geq 0, \\
& d\left(f_{-n}^{-1}\left(x_{n+1}\right), x_{n}\right)<\delta, \quad \text { for } n \leq-1 .
\end{aligned}
$$

For given $\varepsilon>0$, a $\delta$-pseudo orbit $\left\{x_{n}\right\}_{n=-\infty}^{\infty}$ is said to be $\varepsilon$-traced by $y \in X$ if $d\left(F_{n}(y), x_{n}\right)<\varepsilon$ for all $n \in \mathbb{Z}$.

The time varying homeomorphism $F$ is said to have shadowing property or pseudo orbit tracing property (P.O.T.P.) if, for every $\varepsilon>0$, there exists a $\delta>0$ such that every $\delta$ pseudo orbit is $\varepsilon$-traced by some point of $X$.

Remark 19. Note that $\left\{x_{n}\right\}_{n=-\infty}^{\infty}$ in $X$ is a $\delta$-pseudo orbit of $F$ if for $n \geq 1$ we have $d\left(f_{n}\left(x_{n-1}\right), x_{n}\right)<\delta$ and for $n \leq-1$ we have $d\left(f_{n}\left(x_{n-1}\right), x_{n}\right)<\delta$.
Remark 20. If in the above definition $f_{n}=f$, for all $n \geq 0$, where $f: X \rightarrow X$ is homeomorphism, then P.O.T.P. of time varying homeomorphism $F=\left\{f_{n}\right\}_{n=0}^{\infty}$ on $X$ is equivalent to P.O.T.P. of $f$ on $X[20]$.

Remark 21. Note that shadowing property of time varying homeomorphism $F$ is independent of choice of metric if $X$ is compact.

Theorem 22. Let $F=\left\{f_{n}\right\}_{n=0}^{\infty}$ be a time varying homeomorphism on a metric space $\left(X, d_{1}\right)$ uniformly conjugate to time varying homeomorphism $G=\left\{g_{n}\right\}_{n=0}^{\infty}$ on metric space $\left(Y, d_{2}\right)$. Then $F$ has shadowing property if and only if $G$ has shadowing property.

Proof. Given any $\varepsilon>0$, applying the uniform continuity of $h$ implies that there exists $0<\varepsilon_{1}<\varepsilon$ such that, for any $x_{1}, x_{2} \in$ $X$ with $d_{1}\left(x_{1}, x_{2}\right)<\varepsilon_{1}, d_{2}\left(h\left(x_{1}\right), h\left(x_{2}\right)\right)<\varepsilon$. As $F$ has P.O.T.P., there exists $0<\delta_{1}<\varepsilon_{1}$ such that every $\delta_{1}$-pseudo orbit of $F$ is $\varepsilon_{1}$-traced by some point of $X$. Noting the fact that $h^{-1}$ is uniformly continuous, there exists $0<\delta<\delta_{1}$ such that, for any $y_{1}, y_{2} \in Y$ with $d_{2}\left(y_{1}, y_{2}\right)<\delta, d_{1}\left(h^{-1}\left(y_{1}\right), h^{-1}\left(y_{2}\right)\right)<\delta_{1}$.

Now, we assert that every $\delta$-pseudo orbit of $G$ is $\varepsilon$-traced by some point of $Y$.

In fact, for any $\delta$-pseudo orbit $\left\{y_{n}\right\}$ of $G$, applying

$$
\begin{aligned}
& d_{1}\left(f_{n}\left(h^{-1}\left(y_{n}\right)\right), h^{-1}\left(y_{n+1}\right)\right) \\
& =d_{1}\left(h^{-1}\left(g_{n}\left(y_{n}\right)\right), h^{-1}\left(y_{n+1}\right)\right)<\delta_{1},
\end{aligned}
$$

it follows that $\left\{h^{-1}\left(y_{n}\right)\right\}$ is a $\delta_{1}$-pseudo orbit of $F$. Then there exists $x \in X$ such that $\left\{h^{-1}\left(y_{n}\right)\right\}$ is $\varepsilon_{1}$-traced by $x$. This implies that, for any $n \in \mathbb{Z}, d_{2}\left(G_{n}(h(x)), y_{n}\right)=d_{2}\left(h\left(F_{n}(x)\right)\right.$, $\left.h\left(h^{-1}\left(y_{n}\right)\right)\right)<\varepsilon$.

By similar arguments using uniform continuity of $h$ one can prove the converse.

Let $\left(X, d_{1}\right)$ and $\left(Y, d_{2}\right)$ be metric spaces. Define metric $d$ on $X \times Y$ by

$$
\begin{array}{r}
d\left(\left(x_{1}, y_{1}\right),\left(x_{2}, y_{2}\right)\right)=\max \left\{d_{1}\left(x_{1}, x_{2}\right), d_{2}\left(y_{1}, y_{2}\right)\right\}, \\
\left(x_{1}, y_{1}\right),\left(x_{2}, y_{2}\right) \in X \times Y .
\end{array}
$$

By similar arguments given in Theorem 3.2 in [19] we can prove the following result.

Theorem 23. Let $F=\left\{f_{n}\right\}_{n=0}^{\infty}, G=\left\{g_{n}\right\}_{n=0}^{\infty}$ be time varying homeomorphisms. Then $F$ and $G$ have shadowing property if and only if the time varying homeomorphism $F \times G=$ $\left\{f_{n} \times g_{n}\right\}_{n=0}^{\infty}$ has shadowing property on $X \times Y$. Hence every finite direct product of time varying homeomorphisms having shadowing property, has shadowing property.

Theorem 24. Let $F=\left\{f_{n}\right\}_{n=0}^{\infty}$ be the time varying homeomorphism on a metric space $(X, d)$. Then $F$ has P.O.T.P. if and only if $F^{-1}$ has P.O.T.P.

Proof. The proof follows observing that $\left\{x_{n}\right\}$ is a $\delta$-pseudo orbit of $F$ if and only if $\left\{y_{n}=x_{-n}\right\}$ is a $\delta$-pseudo orbit of $F^{-1}$.

Using the above result and similar arguments given in Theorem 3.3 in [19] we get the following result. 
Theorem 25. Let $(X, d)$ be a compact metric space and $\left\{f_{n}\right\}_{n=0}^{\infty}$ an equicontinuous family of self-homeomorphisms on $X$. Then time varying homeomorphism $F=\left\{f_{n}\right\}_{n=0}^{\infty}$ has P.O.T.P. if and only if $F^{k}$ has P.O.T.P. for any $k \in \mathbb{Z}-\{0\}$.

\section{Topological Stability of Nonautonomous Discrete System Induced by a Sequence of Homeomorphisms on a Compact Metric Space}

Let $(X, d)$ be a compact metric space, and define standard bounded metric $d_{1}$ on $X$ by

$$
d_{1}(x, y)=\max \{d(x, y), 1\}, \quad x, y \in X .
$$

And let $(\mathscr{H}(X), \eta)$ be the space of all homeomorphisms on $X$, where metric $\eta$ is defined by

$$
\eta(f, g)=\sup _{x \in X} d_{1}(f(x), g(x)), \quad f, g \in \mathscr{H}(X) .
$$

Let $\mathscr{G}(X)$ be the collection of all time varying homeomorphisms on $X$. We define a metric $\rho$ on $\mathscr{G}(X)$ as follows:

$$
\begin{aligned}
& \text { For } F=\left\{f_{n}\right\}_{n=0}^{\infty} \text { and } G=\left\{g_{n}\right\}_{n=0}^{\infty}, \\
& \rho(F, G)=\max \left\{\sup _{n \geq 0} \eta\left(f_{n}, g_{n}\right), \sup _{n \geq 0} \eta\left(f_{n}^{-1}, g_{n}^{-1}\right)\right\} .
\end{aligned}
$$

Definition 26. A time varying homeomorphism $F$ is said to be topologically stable in $\mathscr{G}(X)$ if for every $\varepsilon>0$ there exists a $\delta, 0<\delta<1$, such that for a time varying homeomorphism $G$ with $\rho(F, G)<\delta$ there is a continuous map $h$ so that, for all $x \in X, d(h(x), x)<\varepsilon$ and $d\left(F_{n}(h(x)), G_{n}(x)\right)<\varepsilon$, for all $n \in \mathbb{Z}$.

Theorem 27. Let $(X, d)$ be a compact metric space and let $F=\left\{f_{n}\right\}_{n=0}^{\infty}$ be a time varying homeomorphism on $X$ which is expansive and has shadowing then $F$ is topologically stable in $\mathscr{G}(X)$.

Proof. Let $e>0$ be an expansive constant for time varying homeomorphism $F=\left\{f_{n}\right\}_{n=0}^{\infty}$. choose $\eta$ such that $0<\eta<$ $e / 3$. Since $F$ has shadowing property therefore for above $\eta$ we get $\delta, 0<\delta<\min \{e / 3,1\}$ be a real number such that every $\delta$-pseudo orbit of $F$ can be $\eta$-traced by some $F$ orbit. Using expansiveness of $F$,one can prove that $\delta$-pseudo orbit is $\eta$ traced by unique $x \in X$.

Let $G=\left\{g_{n}\right\}_{n=0}^{\infty}$ be a time varying map on $X$ such that $\rho(F, G)<\delta$. Let $x \in X$ Since

$$
\begin{array}{ll}
d\left(f_{n}\left(G_{n-1}(x)\right), G_{n}(x)\right) & \\
\quad=d\left(f_{n}\left(G_{n-1}(x)\right), g_{n}\left(G_{n-1}(x)\right)\right)<\delta, & \\
& \quad \forall n \geq 0, \\
d\left(f_{-n}^{-1}\left(G_{n+1}(x)\right), G_{n}(x)\right) & \\
\quad=d\left(f_{-n}^{-1}\left(G_{n}(x)\right), g_{-n}^{-1}\left(G_{n}(x)\right)\right)<\delta, &
\end{array}
$$$$
\forall n<0,
$$

therefore $\left\{G_{n}(x)\right\}_{n=-\infty}^{\infty}$ is a $\delta$-pseudo orbit for $F=\left\{f_{n}\right\}_{n=0}^{\infty}$. Let $h(x) \in X$ be unique element of $X$ whose $F$-orbit $\eta$ traces $\left\{G_{n}(x)\right\}_{n=-\infty}^{\infty}$. So we get a map $h: X \rightarrow X$ with $d\left(F_{n}(h(x)), G_{n}(x)\right)<\varepsilon$, for $n \in \mathbb{Z}$ and $x \in X$. Letting $n=0$, we have $d(h(x), x)<\varepsilon$, for each $x \in X$.

By similar arguments given in Theorem 4.1 in [19] we can prove that $h$ is continuous.

\section{Conflict of Interests}

The authors declare that there is no conflict of interests regarding the publication of this paper.

\section{Acknowledgments}

The second author is supported by UGC Major Research Project F.N. 42-25/2013 (SR) for carrying out this research. The authors thank one of the referees for some useful suggestions.

\section{References}

[1] S. Kolyada and L. Snoha, "Topological entropy of nonautonomous dynamical systems," Random \& Computational Dynamics, vol. 4, no. 2-3, pp. 205-233, 1996.

[2] S. Kolyada, L. Snoha, and S. Trofimchuk, "On minimality of nonautonomous dynamical systems," Nonlinear Oscillations, vol. 7, no. 1, pp. 86-92, 2004.

[3] R. Kempf, "On $\Omega$-limit sets of discrete-time dynamical systems," Journal of Difference Equations and Applications, vol. 8, no. 12, pp. 1121-1131, 2002.

[4] J. S. Cánovas, "On $\omega$-limit sets of non-autonomous discrete systems," Journal of Difference Equations and Applications, vol. 12, no. 1, pp. 95-100, 2006.

[5] W. Krabs, "Stability and controllability in non-autonomous time-discrete dynamical systems," Journal of Difference Equations and Applications, vol. 8, no. 12, pp. 1107-1118, 2002.

[6] X. Huang, X. Wen, and F. Zeng, "Topological pressure of nonautonomous dynamical systems," Nonlinear Dynamics and Systems Theory, vol. 8, no. 1, pp. 43-48, 2008.

[7] X. Huang, X. Wen, and F. Zeng, "Pre-image entropy of nonautonomous dynamical systems," Journal of Systems Science \& Complexity, vol. 21, no. 3, pp. 441-445, 2008.

[8] F. Balibrea and P. Oprocha, "Weak mixing and chaos in nonautonomous discrete systems," Applied Mathematics Letters, vol. 25, no. 8, pp. 1135-1141, 2012.

[9] J. S. Cánovas, "Li-Yorke chaos in a class of nonautonomous discrete systems," Journal of Difference Equations and Applications, vol. 17, no. 4, pp. 479-486, 2011.

[10] J. S. Cánovas, "Recent results on non-autonomous discrete systems," Boletín de la Sociedad Española de Matemática Aplicada, no. 51, pp. 33-40, 2010.

[11] J. Dvořáková, "Chaos in nonautonomous discrete dynamical systems," Communications in Nonlinear Science and Numerical Simulation, vol. 17, no. 12, pp. 4649-4652, 2012.

[12] P. Oprocha and P. Wilczyński, "Chaos in nonautonomous dynamical systems," Analele Stiintifice ale Universitatii Ovidius Constanta, Seria Matematica, vol. 17, no. 3, pp. 209-221, 2009. 
[13] Y. Shi and G. Chen, "Chaos of time-varying discrete dynamical systems," Journal of Difference Equations and Applications, vol. 15, no. 5, pp. 429-449, 2009.

[14] C. Tian and G. Chen, "Chaos of a sequence of maps in a metric space," Chaos, Solitons and Fractals, vol. 28, no. 4, pp. 1067-1075, 2006.

[15] X. Wu and P. Zhu, "Chaos in a class of non-autonomous discrete systems," Applied Mathematics Letters, vol. 26, no. 4, pp. 431436, 2013.

[16] L. Liu and B. Chen, "On $\omega$-limit sets and attraction of nonautonomous discrete dynamical systems," Journal of the Korean Mathematical Society, vol. 49, no. 4, pp. 703-713, 2012.

[17] M. Murillo-Arcila and A. Peris, "Mixing properties for nonautonomous linear dynamics and invariant sets," Applied Mathematics Letters, vol. 26, no. 2, pp. 215-218, 2013.

[18] K. Yokoi, "Recurrence properties of a class of nonautonomous discrete systems," Bulletin of the Belgian Mathematical Society. Simon Stevin, vol. 20, no. 4, pp. 689-705, 2013.

[19] D. Thakkar and R. Das, "Topological stability of a sequence of maps on a compact metric space," Bulletin of Mathematical Sciences, vol. 4, no. 1, pp. 99-111, 2014.

[20] N. Aoki and K. Hiraide, Topological Theory of Dynamical Systems, North-Holland, Amsterdam, The Netherlands, 1994.

[21] B. F. Bryant, "Expansive self-homeomorphisms of a compact metric space," The American Mathematical Monthly, vol. 69, no. 5, pp. 386-391, 1962.

[22] H. B. Keynes and J. B. Robertson, "Generators for topological entropy and expansiveness," Mathematical Systems Theory, vol. 3, pp. 51-59, 1969. 


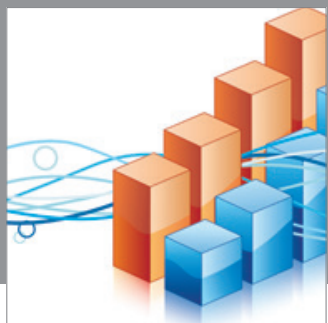

Advances in

Operations Research

mansans

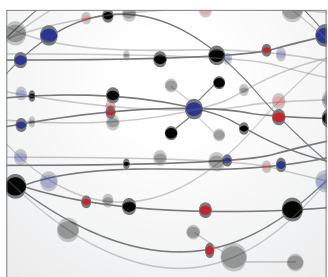

The Scientific World Journal
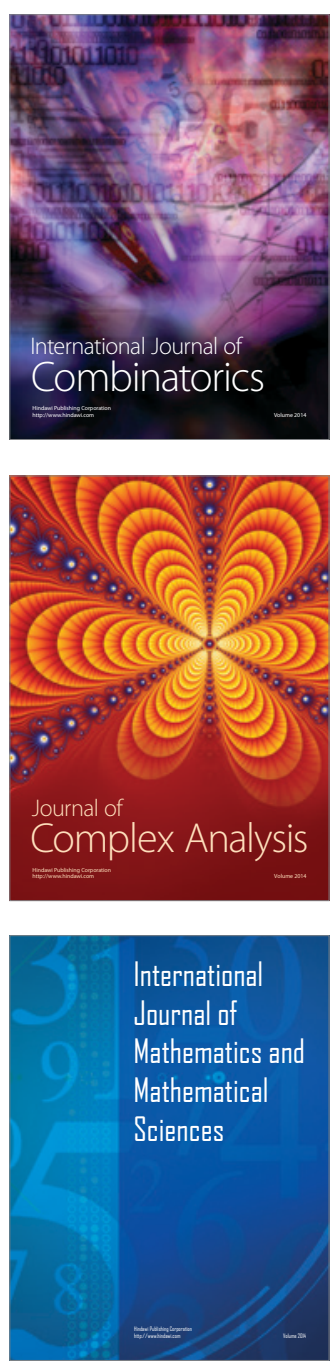
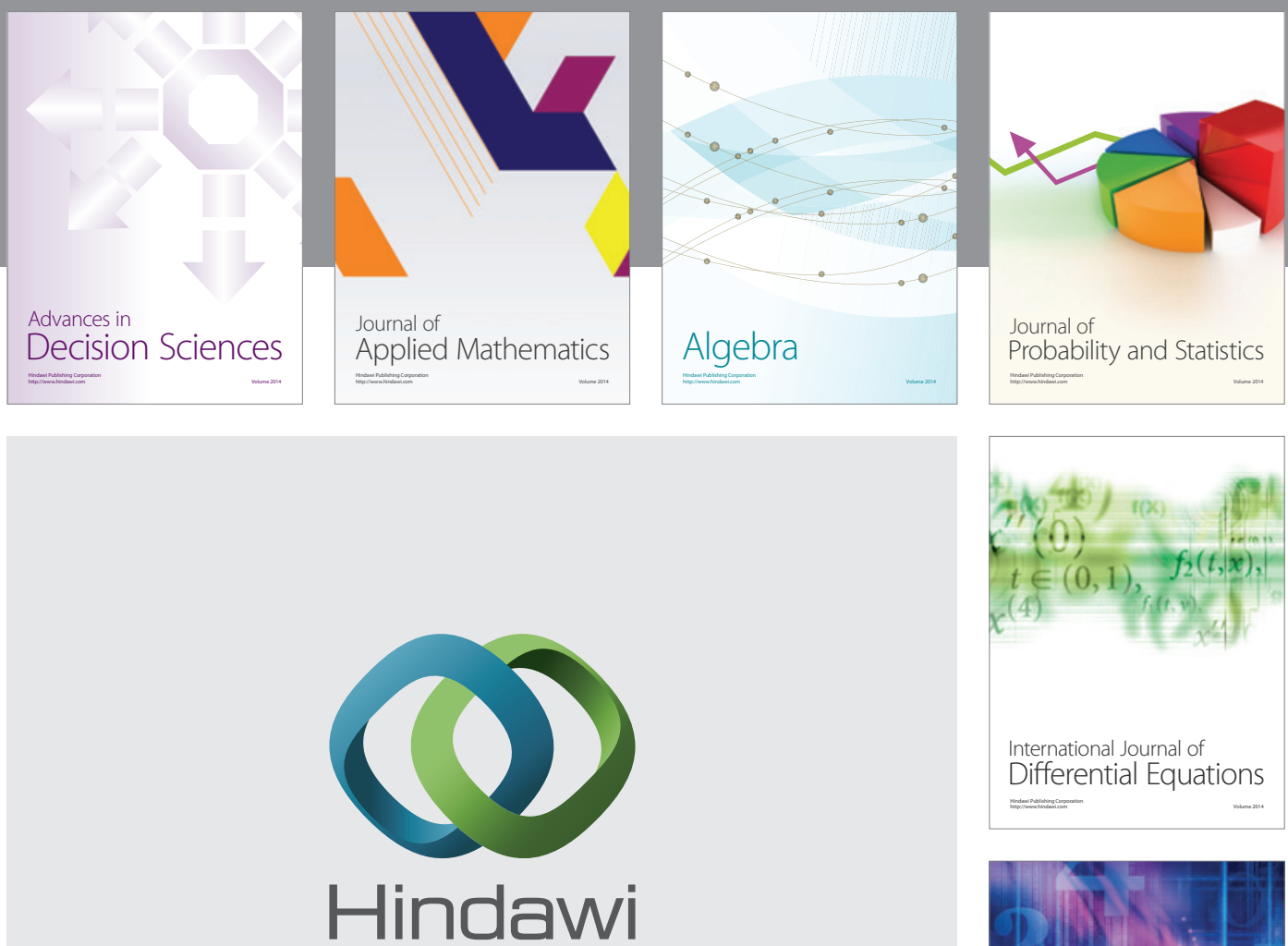

Submit your manuscripts at http://www.hindawi.com
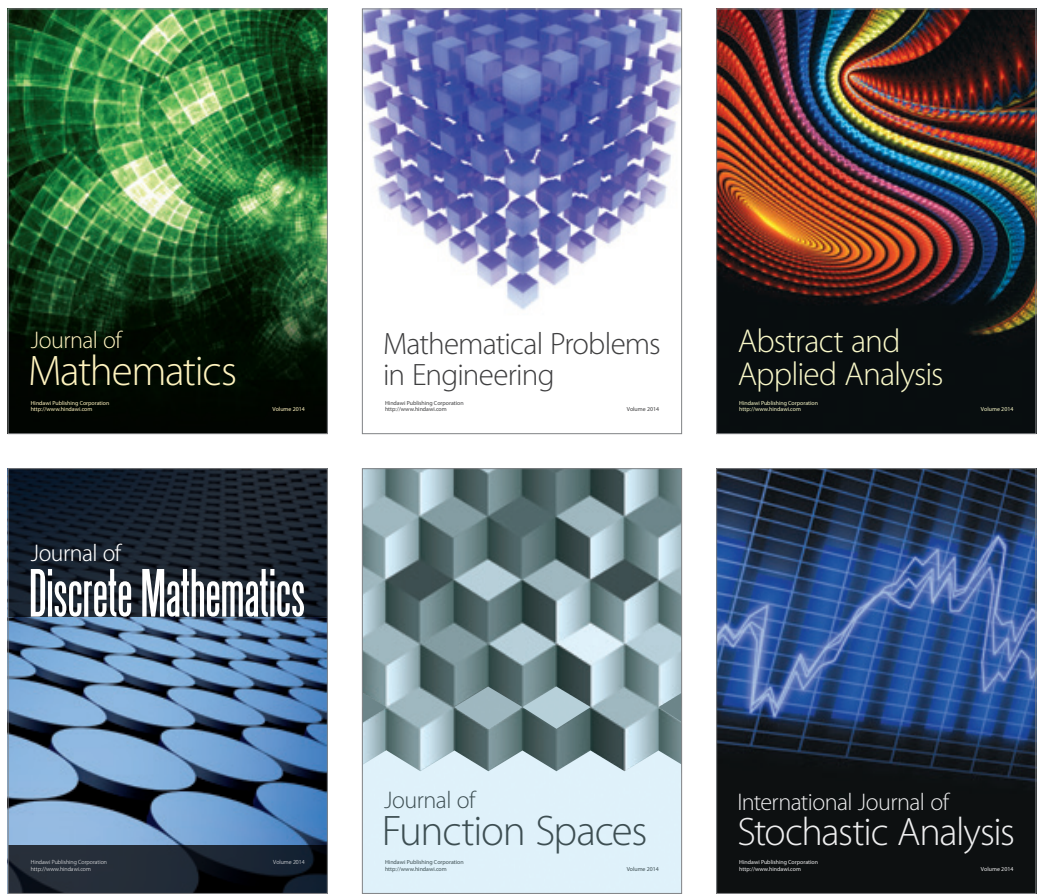

Journal of

Function Spaces

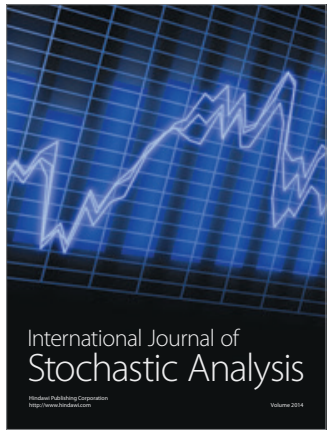

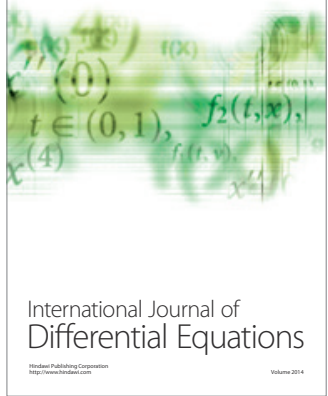
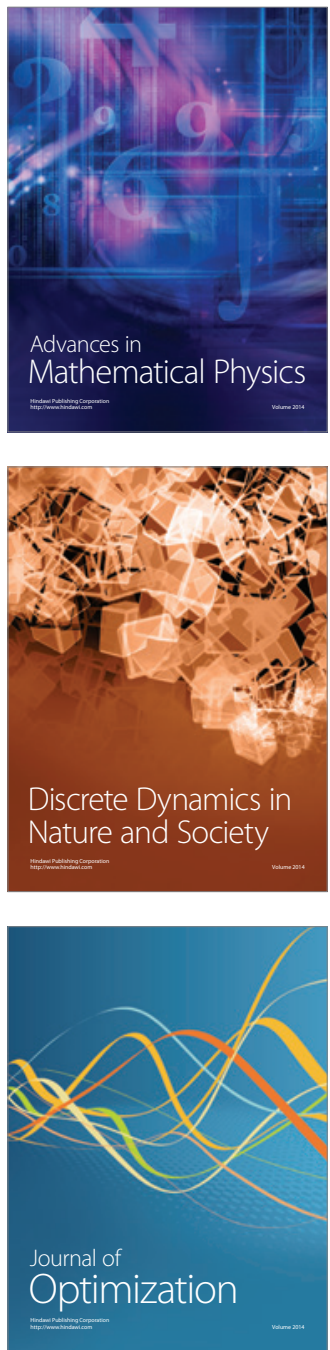